\title{
Phenotypic variability of cowpea genotypes for immature seed harvesting ${ }^{1}$
}

\author{
Deisy Aiane Lima de Aquino ${ }^{2}$, Carlos Antônio Fernandes Santos ${ }^{3}$, Danillo Olegário Matos da Silva ${ }^{4}$
}

\section{ABSTRACT}

Seed color, brightness and texture are important aspects for the consumption of immature beans. This study aimed to estimate the phenotypic variability among thirty cowpea genotypes for immature seed harvesting, in order to guide breeding programs. The experiment was carried out in a randomized blocks design, with three replications. The evaluated traits were immature seeds yield, pods and dry seeds production, number of immature pods, 100-immature seeds weight, immature pods length, number of seeds per immature pod, cooking time, harvesting period of immature pods, seed color, pod color, plant growth habit, opening of immature pods, seed threshing from immature pods, cultivation value and seed browning after threshing. Anova and three clustering methods were applied: UPGMA using the Jaccard coefficient of similarity; modified Tocher method based on the Gower algorithm; and k-means. All the analyses were performed based on the variable means of experiments evaluated in six environments. A statistically significant phenotypic variability was observed among all the quantitative traits. Discrepancies occurred in the accessions grouping and number of clusters. The accession BRS Acauã, in one group, and P508 and PCCR3F6L15, in other groups, showed a good quality for immature grain harvesting, were consistently grouped into different clusters and are recommended for use as parents in the development of new cultivars.

KEYWORDS: Vigna unguiculata, cluster analysis, genetic variability.

\section{INTRODUCTION}

In northeastern Brazil, cowpea [Vigna unguiculata (L.) Walp] is mainly grown for dry seed production, although the commercialization

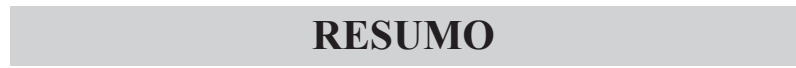

Variabilidade fenotípica de genótipos de feijão-caupi destinados à colheita de sementes imaturas

Cor, brilho e textura das sementes são importantes para o consumo de feijão imaturo. Objetivou-se estimar a variabilidade fenotípica entre trinta genótipos de feijão-caupi destinados à colheita de sementes imaturas, para orientar programas de melhoramento. O experimento foi conduzido em delineamento de blocos casualizados, com três repetições. As características avaliadas foram rendimento de sementes imaturas, produção de vagens e sementes secas, número de vagens imaturas, peso de 100 sementes imaturas, comprimento de vagens imaturas, número de sementes por vagem imatura, tempo de cozimento, período de colheita de vagens imaturas, cor da semente, cor da vagem, hábito de crescimento da planta, abertura de vagens imaturas, debulha de sementes de vagens imaturas, valor de cultivo e escurecimento das sementes após a debulha. A Anova e três análises de agrupamento foram adotadas: UPGMA, com o coeficiente de similaridade de Jaccard; método Tocher modificado, com o algoritmo de Gower; e k-means. Todas as análises foram realizadas com base em médias das variáveis de experimentos avaliados em seis ambientes. Variabilidade fenotípica estatisticamente significativa foi observada entre todas as variáveis quantitativas. Discrepâncias ocorreram no agrupamento de acessos e número de agrupamentos. O acesso BRS Acauã, em um grupo, e P508 e PCCR3F6L15, em outros grupos, apresentaram boa qualidade para colheita imatura, foram consistentemente agrupados em diferentes grupos e são indicados para utilização como genitores no desenvolvimento de novas cultivares.

PALAVRAS-CHAVE: Vigna unguiculata, análise de agrupamento, variabilidade genética.

of immature seeds is also expressive. Immature seeds are obtained from pods around maturity, i.e., immediately before or shortly after the plant stops accumulating photosynthates and begins the natural dehydration process, which occurs with

${ }^{1}$ Received: July 09, 2021. Accepted: Nov. 04, 2021. Published: Nov. 26, 2021. DOI: 10.1590/1983-40632021v5169614.

${ }^{2}$ Universidade Estadual de Feira de Santana, Feira de Santana, BA, Brasil.

E-mail/ORCID: deisylima_17@hotmail.com/0000-0001-8897-320X.

${ }^{3}$ Empresa Brasileira de Pesquisa Agropecuária (Embrapa Semiárido), Petrolina, PE, Brasil.

E-mail/ORCID: carlos-fernandes.santos@embrapa.br/0000-0002-6932-6805.

${ }^{4}$ Centro Universitário Maurício de Nassau, Petrolina, PE, Brasil. E-mail/ORCID: danilloolegario@gmail.com/0000-0002-4874-4880. 
seed moisture close to 60 or $70 \%$ (Sousa et al. 2015).

Cowpea has a high genetic variability for almost all morphological and agronomic traits, though little has been investigated in this regard (Teixeira et al. 2010). However, information on the phenotypic variability of cowpea germplasm and on how much of it is due to genetic differences is fundamental in breeding programs (Cruz et al. 2004). According to Cruz et al. (2004), diversity denotes the genetic distance within species, populations or accessions measured through morphological, agronomic, biochemical, physiological and molecular traits.

In the Rio Grande do Norte state (Brazil), Silva Filho et al. (2013) evaluated the morphological traits of dry cowpea seeds and observed variability, highlighting the selection potential of several accessions that can be incorporated into breeding programs. Moreover, Andrade (2010) studied immature seed harvesting in 20 cowpea accessions and found genetic variability for number of days to flowering, number of days to immature pod maturity, immature pod length, cooking time and other traits related to immature seeds. However, an extensive characterization of cowpea germplasm for immature seed harvesting has not been conducted to date in the São Francisco Valley region, to assist in developing cowpea genotypes suitable for cultivation. Therefore, this study aimed to estimate the phenotypic variability of cowpea genotypes for immature seed harvesting and select accessions with traits of agronomic interest for breeding programs in the semi-arid region of Brazil.

\section{MATERIAL AND METHODS}

Thirty cowpea genotypes were selected to evaluate the crop phenotypic variability, including landraces, cultivars and some breeding lines with potential for immature seed harvesting. These genotypes consisted of 14 lines provided by the Embrapa Semiárido, six commercial cultivars and ten landraces collected in cowpea-producing areas of Juazeiro (Bahia state) and Petrolina (Pernambuco state), Brazil (Table 1). The experiments were conducted in experimental fields located in Bebedouro (Petrolina) and Mandacaru (Juazeiro), during the second half of 2013, 2014 and 2015.

A randomized blocks design, with three replications, was used. The plots measured $6 \mathrm{~m}^{2}$ and had two rows spaced $1.0 \mathrm{~m}$ and plants spaced $0.1 \mathrm{~m}$, representing a population of 100,000 plants $\mathrm{ha}^{-1}$. As a common practice in the region, soil fertilization did not occur. Moreover, a micro-sprinkler system was used for irrigation twice a week, to avoid soil waterlogging. Weeding and pest control with insecticides were performed whenever necessary.

The evaluated agronomic traits were immature seed yield $\left(\mathrm{kg} \mathrm{ha}^{-1}\right)$, dry seed yield $\left(\mathrm{kg} \mathrm{ha}^{-1}\right)$, immature pod yield $\left(\mathrm{kg} \mathrm{ha}^{-1}\right)$, number of immature pods, 100-immature seed weight (g), immature pod length $(\mathrm{cm})$, number of seeds per immature pod, cooking time (Matteson ${ }^{\circledR}$ cooker; min) and harvesting period of immature pods (days).

The evaluated morphological traits were seed and pod color $(1=$ cream; 2 = green; 3 = purple); plant growth habit $(1=$ erect; $2=$ semi-erect; $3=$ semi-prostrate; 4 = prostrate); opening of immature pods by manual threshing and seed release from immature pods by manual threshing $(1=$ very difficult; 2 = difficult; 3 = easy; $4=$ very easy); cultivation value considering the appropriate traits for commercial cultivation $(1=$ none - inappropriate; $2=$ little - poor; 3 = many - adequate; 4 = most - good; $5=$ all - very good; $6=$ all - excellent); and seed browning at threshing and 24 and $48 \mathrm{~h}$ after threshing $(1=$ light; 2 = little dark; 3 = medium dark; $4=$ dark; $5=$ very dark).

The analysis of variance (Anova) of the six experiments considered only the agronomic traits, and all means were grouped by the Scott-Knott test at $5 \%$ of probability, using the Genes software (Cruz 2016). The means of immature seed yield, dry seed yield and immature pod yield for each environment were adjusted to the mean number of plants in the experimental units using the covariance method with the aid of a SAS code (Vencovsky \& Barriga 1992).

The means of the cowpea genotypes were subjected to three cluster analyses: unweighted pair group method with arithmetic mean (UPGMA), using the NTSYs software (Rohlf 2000), considering the Jaccard similarity coefficient with nine quantitative variables categorized into five classes; presence (1) or absence $(0)$ of traits evaluated in each class using the modified Tocher method based on the Gower algorithm with the Genes software (Cruz 2016); $\mathrm{k}$-means clustering with qualitative data transformed to $\sqrt{\mathrm{x}}+1$, considering the formation of three groups (cultivars, lines and varieties from local producers). 
Table 1. Origin of the cowpea genotypes [Vigna unguiculata (L.) Walp] evaluated for immature seed harvesting [Pernambuco (PE) and Bahia (BA) states, Brazil].

\begin{tabular}{|c|c|c|}
\hline Treatments & Genotypes & Sample origin \\
\hline 1 & BRS-Acauã & Embrapa Semiárido - Petrolina, PE \\
\hline 2 & BRS-Guariba & Embrapa Semiárido - Petrolina, PE \\
\hline 3 & BRS-Marataoã & Embrapa Semiárido - Petrolina, PE \\
\hline 4 & BRS-Patativa & Embrapa Semiárido - Petrolina, PE \\
\hline 5 & BRS-Pujante & Embrapa Semiárido - Petrolina, PE \\
\hline 6 & BRS-Rouxinol & Embrapa Semiárido - Petrolina, PE \\
\hline 7 & Line PC951015D01E & Embrapa Semiárido - Petrolina, PE \\
\hline 8 & Line PC950409D02E & Embrapa Semiárido - Petrolina, PE \\
\hline 9 & Line PC951016D01E & Embrapa Semiárido - Petrolina, PE \\
\hline 10 & Line CPCR3F6L15 & Embrapa Semiárido - Petrolina, PE \\
\hline 11 & Line CPCR3F6L17 & Embrapa Semiárido - Petrolina, PE \\
\hline 12 & Line C1J & Embrapa Semiárido - Petrolina, PE \\
\hline 13 & Line $\mathrm{C} 2 \mathrm{M}$ & Embrapa Semiárido - Petrolina, PE \\
\hline 14 & Line $\mathrm{C} 2 \mathrm{~S}$ & Embrapa Semiárido - Petrolina, PE \\
\hline 15 & Line $\mathrm{C} 3 \mathrm{~F}$ & Embrapa Semiárido - Petrolina, PE \\
\hline 16 & Line C3Q & Embrapa Semiárido - Petrolina, PE \\
\hline 17 & Line C3S & Embrapa Semiárido - Petrolina, PE \\
\hline 18 & Line P290 & Embrapa Semiárido - Petrolina, PE \\
\hline 19 & Line P303 & Embrapa Semiárido - Petrolina, PE \\
\hline 20 & Line P508 & Embrapa Semiárido - Petrolina, PE \\
\hline 21 & PJJ21 & Nilo Coelho N8 - Petrolina, PE \\
\hline 22 & PJM22 & Nilo Coelho - Petrolina, PE \\
\hline 23 & PL23 & Mandacaru - Juazeiro, BA \\
\hline 24 & PAG24 & Maniçoba - Juazeiro, BA \\
\hline 25 & PC25 & Nilo Coelho N9 - Petrolina, PE \\
\hline 26 & PD26 & Maniçoba - Juazeiro, BA \\
\hline 27 & PJ27 & Maniçoba - Juazeiro, BA \\
\hline 28 & PJJ28 & Nilo Coelho N8 - Petrolina, PE \\
\hline 29 & PJN29 & Nilo Coelho - Petrolina, PE \\
\hline 30 & PLP30 & Mandacaru - Juazeiro, BA \\
\hline
\end{tabular}

The k-means analysis was performed using the fastclus procedure, which employs the nearest centroid sorting method, and the Euclidean distance to evaluate the dissimilarity of the means (SAS Institute 1989).

\section{RESULTS AND DISCUSSION}

Seed and pod color were different, with a predominant cream color for seed color. The pod color of the genotypes BRS-Guariba, PC951016D01E, CPCR3F6L15, C1J, C2S, C3S and P303 was purple. In contrast, the genotypes PC951015D01E, P508, PJM22 and PL23 had a cream pod color, while the remaining genotypes were green (Table 2). The landraces had semi-prostrate and prostrate plant growth habits, while the cowpea lines and cultivars from research institutes had semi-erect and prostrate plant growth habits (Table 2).
The genotypes differed with regard to the opening of immature pods and seed release from immature pods by manual threshing. The genotypes BRS-Acauã, C3Q, PJJ21 and PD26 showed an easier opening of immature pods and seed release from immature pods (Table 2). The cultivation values of all genotypes were close to the maximum value required for commercial cultivation, except for the genotype $\mathrm{C} 3 \mathrm{~F}$, which showed plants with many appropriate traits (Table 2). The seed browning after threshing showed differences among all the genotypes, with the BRS-Acauã and PJN29 showing the lowest seed browning at $48 \mathrm{~h}$ after threshing (Table 2).

The cowpea genotypes showed statistically significant differences by the F-test $(\mathrm{p}<0.05)$, suggesting a high phenotypic variability for all the agronomic variables. The immature seed yield of the cowpea genotypes ranged from 931 (BRS-Marataoã) to 2,532 $\mathrm{kg} \mathrm{ha}^{-1}$ (P508) (Table 3). On the other hand, 
Table 2. Means of nine morphological traits of thirty cowpea genotypes [Vigna unguiculata(L.) Walp] evaluated for immature seed harvesting.

\begin{tabular}{|c|c|c|c|c|c|c|c|c|c|}
\hline Accession & $\mathrm{SC}$ & $\mathrm{PC}$ & PGH & OIP & SRP & $\mathrm{CV}$ & SB0h & SB24h & SB48h \\
\hline BRS-Acauã & $1.2 \mathrm{~d}$ & $2.4 \mathrm{c}$ & $2.5 \mathrm{~d}$ & $3.1 \mathrm{a}$ & $3.3 \mathrm{a}$ & $3.5 \mathrm{~d}$ & $1.0 \mathrm{a}$ & $1.6 \mathrm{f}$ & $2.4 \mathrm{e}$ \\
\hline BRS-Guariba & $1.4 \mathrm{c}$ & $2.9 \mathrm{a}$ & $2.7 \mathrm{c}$ & $2.7 \mathrm{c}$ & $2.6 \mathrm{c}$ & $3.8 \mathrm{c}$ & $1.0 \mathrm{c}$ & $2.1 \mathrm{e}$ & $2.7 \mathrm{~d}$ \\
\hline BRS-Marataoã & $1.2 \mathrm{~d}$ & $2.2 \mathrm{c}$ & $2.3 \mathrm{e}$ & $2.7 \mathrm{c}$ & $2.9 \mathrm{~b}$ & $3.8 \mathrm{c}$ & $1.0 \mathrm{c}$ & $1.7 \mathrm{f}$ & $2.8 \mathrm{~d}$ \\
\hline BRS-Patativa & $1.4 \mathrm{c}$ & $1.9 \mathrm{~d}$ & $3.2 \mathrm{a}$ & $2.6 \mathrm{c}$ & $2.6 \mathrm{c}$ & $4.0 \mathrm{~b}$ & $1.0 \mathrm{c}$ & $2.5 \mathrm{~d}$ & $3.3 \mathrm{c}$ \\
\hline BRS-Pujante & $1.0 \mathrm{~d}$ & $1.7 \mathrm{e}$ & $3.0 \mathrm{~b}$ & $2.9 \mathrm{~b}$ & $2.9 \mathrm{~b}$ & $3.5 \mathrm{~d}$ & $1.0 \mathrm{c}$ & $2.1 \mathrm{e}$ & $2.9 \mathrm{~d}$ \\
\hline BRS-Rouxinol & $1.1 \mathrm{~d}$ & $1.7 \mathrm{e}$ & $2.5 \mathrm{~d}$ & $2.6 \mathrm{c}$ & $2.6 \mathrm{c}$ & $3.8 \mathrm{c}$ & $1.0 \mathrm{c}$ & $2.6 \mathrm{c}$ & $3.2 \mathrm{c}$ \\
\hline PC951015D01E & $1.1 \mathrm{~d}$ & $1.2 \mathrm{f}$ & $2.7 \mathrm{c}$ & $2.5 \mathrm{c}$ & $2.4 \mathrm{c}$ & $4.0 \mathrm{~b}$ & $1.0 \mathrm{c}$ & $1.7 \mathrm{e}$ & $2.8 \mathrm{~d}$ \\
\hline PC950409D02E & $1.5 \mathrm{~b}$ & $1.9 \mathrm{~d}$ & $2.8 \mathrm{c}$ & $2.6 \mathrm{c}$ & $2.4 \mathrm{c}$ & $3.7 \mathrm{c}$ & $1.0 \mathrm{c}$ & $3.2 \mathrm{~b}$ & $3.9 \mathrm{~b}$ \\
\hline PC951016D01E & $1.1 \mathrm{~d}$ & $2.8 \mathrm{a}$ & $3.0 \mathrm{~b}$ & $2.6 \mathrm{c}$ & $2.6 \mathrm{c}$ & $4.0 \mathrm{~b}$ & $1.0 \mathrm{c}$ & $2.6 \mathrm{c}$ & $3.4 \mathrm{c}$ \\
\hline CPCR3F6L15 & $1.1 \mathrm{~d}$ & $1.7 \mathrm{e}$ & $2.0 \mathrm{f}$ & $2.6 \mathrm{c}$ & $2.8 \mathrm{~b}$ & $4.2 \mathrm{a}$ & $1.0 \mathrm{c}$ & $2.1 \mathrm{e}$ & $3.2 \mathrm{c}$ \\
\hline CPCR3F6L17 & $1.1 \mathrm{~d}$ & $3.0 \mathrm{a}$ & $3.0 \mathrm{~b}$ & $2.9 \mathrm{~b}$ & $2.7 \mathrm{c}$ & $3.5 \mathrm{~d}$ & $1.0 \mathrm{c}$ & $2.2 \mathrm{e}$ & $3.1 \mathrm{~b}$ \\
\hline $\mathrm{C} 1 \mathrm{~J}$ & $1.2 \mathrm{~d}$ & $3.0 \mathrm{a}$ & $3.0 \mathrm{~b}$ & $2.6 \mathrm{~b}$ & $2.6 \mathrm{c}$ & $4.0 \mathrm{~b}$ & $1.1 \mathrm{~b}$ & $2.8 \mathrm{c}$ & $3.7 \mathrm{~b}$ \\
\hline $\mathrm{C} 2 \mathrm{M}$ & $1.6 \mathrm{~b}$ & $1.8 \mathrm{e}$ & $2.7 \mathrm{c}$ & $2.4 \mathrm{c}$ & $2.7 \mathrm{c}$ & $3.7 \mathrm{c}$ & $1.0 \mathrm{c}$ & $2.0 \mathrm{e}$ & $3.2 \mathrm{c}$ \\
\hline $\mathrm{C} 2 \mathrm{~S}$ & $1.2 \mathrm{~d}$ & $2.8 \mathrm{e}$ & $3.0 \mathrm{~b}$ & $2.7 \mathrm{c}$ & $2.7 \mathrm{c}$ & $3.8 \mathrm{c}$ & $1.0 \mathrm{c}$ & $2.5 \mathrm{~d}$ & $3.2 \mathrm{c}$ \\
\hline $\mathrm{C} 3 \mathrm{~F}$ & $1.1 \mathrm{~d}$ & $1.7 \mathrm{a}$ & $2.5 \mathrm{~d}$ & $2.9 \mathrm{~b}$ & $2.9 \mathrm{~b}$ & $3.3 \mathrm{e}$ & $1.6 \mathrm{a}$ & $3.7 \mathrm{a}$ & $4.3 \mathrm{a}$ \\
\hline C3Q & $1.4 \mathrm{c}$ & $1.8 \mathrm{e}$ & $2.5 \mathrm{~d}$ & $3.3 \mathrm{a}$ & $3.3 \mathrm{a}$ & $3.5 \mathrm{~d}$ & $1.2 \mathrm{~b}$ & $2.2 \mathrm{e}$ & $2.9 \mathrm{~d}$ \\
\hline $\mathrm{C} 3 \mathrm{~S}$ & $1.1 \mathrm{~d}$ & $2.7 \mathrm{e}$ & $2.8 \mathrm{c}$ & $2.7 \mathrm{c}$ & $2.5 \mathrm{c}$ & $3.3 \mathrm{e}$ & $1.1 \mathrm{~b}$ & $3.6 \mathrm{a}$ & $4.3 \mathrm{a}$ \\
\hline P290 & $1.0 \mathrm{~d}$ & $1.9 \mathrm{~d}$ & $3.0 \mathrm{~b}$ & $2.6 \mathrm{c}$ & $2.7 \mathrm{c}$ & $3.7 \mathrm{c}$ & $1.1 \mathrm{~b}$ & $2.6 \mathrm{c}$ & $3.9 \mathrm{~b}$ \\
\hline P303 & $1.0 \mathrm{~d}$ & $2.8 \mathrm{a}$ & $3.0 \mathrm{~b}$ & $2.5 \mathrm{c}$ & $2.5 \mathrm{c}$ & $4.0 \mathrm{~b}$ & $1.1 \mathrm{~b}$ & $2.7 \mathrm{c}$ & $3.6 \mathrm{~b}$ \\
\hline P508 & $1.2 \mathrm{~d}$ & $1.3 \mathrm{~d}$ & $2.8 \mathrm{c}$ & $2.8 \mathrm{~b}$ & $2.9 \mathrm{~b}$ & $3.7 \mathrm{c}$ & $1.0 \mathrm{c}$ & $2.4 \mathrm{~d}$ & $3.2 \mathrm{c}$ \\
\hline PJJ21 & $1.3 \mathrm{c}$ & $2.6 \mathrm{~b}$ & $3.2 \mathrm{a}$ & $3.3 \mathrm{a}$ & $3.1 \mathrm{a}$ & $4.0 \mathrm{~b}$ & $1.1 \mathrm{~b}$ & $2.8 \mathrm{c}$ & $3.6 \mathrm{~b}$ \\
\hline PJM22 & $1.2 \mathrm{~d}$ & $1.3 \mathrm{f}$ & $2.7 \mathrm{c}$ & $2.8 \mathrm{~b}$ & $2.7 \mathrm{c}$ & $3.8 \mathrm{c}$ & $1.0 \mathrm{c}$ & $3.3 \mathrm{~b}$ & $4.3 \mathrm{a}$ \\
\hline PL23 & $1.4 \mathrm{c}$ & $1.3 \mathrm{f}$ & $3.3 \mathrm{a}$ & $2.3 \mathrm{c}$ & $2.4 \mathrm{c}$ & $3.7 \mathrm{c}$ & $1.0 \mathrm{c}$ & $2.7 \mathrm{c}$ & $3.2 \mathrm{c}$ \\
\hline PAG24 & $1.2 \mathrm{~d}$ & $1.6 \mathrm{e}$ & $2.8 \mathrm{c}$ & $2.4 \mathrm{c}$ & $2.6 \mathrm{c}$ & $3.8 \mathrm{c}$ & $1.0 \mathrm{c}$ & $2.3 \mathrm{~d}$ & $3.4 \mathrm{c}$ \\
\hline PC25 & $2.7 \mathrm{a}$ & $2.3 \mathrm{c}$ & $2.5 \mathrm{~d}$ & $2.4 \mathrm{c}$ & $3.1 \mathrm{a}$ & $4.0 \mathrm{~b}$ & $1.0 \mathrm{c}$ & $2.8 \mathrm{c}$ & $3.8 \mathrm{~b}$ \\
\hline PD26 & $1.1 \mathrm{~d}$ & $1.7 \mathrm{e}$ & $3.0 \mathrm{~b}$ & $3.1 \mathrm{a}$ & $2.9 \mathrm{~b}$ & $3.8 \mathrm{c}$ & $1.0 \mathrm{c}$ & $3.6 \mathrm{a}$ & $4.1 \mathrm{a}$ \\
\hline РJ27 & $1.3 \mathrm{c}$ & $1.8 \mathrm{e}$ & $2.3 \mathrm{e}$ & $2.5 \mathrm{c}$ & $2.6 \mathrm{c}$ & $3.8 \mathrm{c}$ & $1.0 \mathrm{c}$ & $2.6 \mathrm{c}$ & $3.4 \mathrm{c}$ \\
\hline PJJ28 & $1.1 \mathrm{~d}$ & $2.1 \mathrm{~d}$ & $3.0 \mathrm{~b}$ & $2.4 \mathrm{c}$ & $2.4 \mathrm{c}$ & $3.8 \mathrm{c}$ & $1.0 \mathrm{c}$ & $2.4 \mathrm{~d}$ & $3.4 \mathrm{c}$ \\
\hline PJN29 & $1.3 \mathrm{c}$ & $2.4 \mathrm{c}$ & $2.2 \mathrm{e}$ & $2.6 \mathrm{c}$ & $2.6 \mathrm{c}$ & $3.8 \mathrm{c}$ & $1.0 \mathrm{c}$ & $1.5 \mathrm{f}$ & $1.9 \mathrm{f}$ \\
\hline PLP30 & $1.6 \mathrm{~b}$ & $2.9 \mathrm{a}$ & $3.2 \mathrm{a}$ & $2.9 \mathrm{~b}$ & $2.9 \mathrm{~b}$ & $3.8 \mathrm{c}$ & $1.0 \mathrm{c}$ & $3.0 \mathrm{c}$ & $3.7 \mathrm{~b}$ \\
\hline Mean & 1.5 & 1.74 & 1.9 & 1.8 & 1.9 & 2.18 & 1.4 & 1.8 & 2.05 \\
\hline
\end{tabular}

the BRS-Guariba showed the highest immature seed yield $\left(2,029 \mathrm{~kg} \mathrm{ha}^{-1}\right)$ among the cultivars, whereas, among the cowpea lines, P508, PCCR3F6L15 and P303 showed the highest immature seed yield (Table 3). The immature pod yield ranged from 1,783 (genotype PD26) to 4,715 $\mathrm{kg} \mathrm{ha}^{-1}$ (genotype P508) (Table 3).

The immature pod yield values of the genotypes PC951015D01E, PC950409D02E, PCCR3FL15 and P508 were higher than $3,700 \mathrm{~kg} \mathrm{ha}^{-1}$. Furthermore, the dry seed yield ranged from 557 (genotype PLP30) to $1,350 \mathrm{~kg} \mathrm{ha}^{-1}$ (genotype P508). The genotypes BRSPatativa, PCCR3F6L15 and PJM22 showed dry seed yield values higher than $1,200 \mathrm{~kg} \mathrm{ha}^{-1}$. On the other hand, the cooking time in a Matteson ${ }^{\circledR}$ cooker ranged from 10 to $15.3 \mathrm{~min}$ for the genotypes PLP30, BRSMarataoã and C3Q (Table 3). In addition, the cowpea genotypes PD26, BRS-Pujante, BRS-Rouxinol, C2M, PJM22 and PL23 showed immature pod length values higher than $20 \mathrm{~cm}$. On the other hand, the harvesting period of immature pods ranged from 15.6 to 22.6 days, and the genotypes BRS-Acauã, PCCR3F6F15, C2M and C3F showed the longest harvesting period of immature pods (Table 3 ).

The cowpea cultivars, lines and landraces formed a large group of 24 genotypes by the k-means clustering (Table 4). However, another large group consisting of 19 genotypes was formed when the Tocher method was used (Table 5). All 19 genotypes of group 1, formed using the Tocher method, were clustered in group 2 using the k-means method. However, the cowpea lines $\mathrm{C} 3 \mathrm{~F}$ and $\mathrm{C} 3 \mathrm{~S}$ and the genotype PD26 were clustered in the same group by the two methods, while the other cowpea genotypes 
Table 3. Mean square of treatments (MST), coefficient of variation (CV) and means of nine agronomic variables of thirty cowpea genotypes [Vigna unguiculata (L.) Walp] evaluated for immature seed harvesting.

\begin{tabular}{|c|c|c|c|c|c|c|c|c|c|}
\hline Accession & ISY & IPY & DSY & NIP & $100 \mathrm{SW}$ & $\mathrm{CT}$ & IPL & NSP & HPP \\
\hline BRS-Acauã & $1,986 \mathrm{c}$ & $3,388 \mathrm{~b}$ & $1,209 \mathrm{a}$ & $269.5 \mathrm{a}$ & $45.4 \mathrm{a}$ & $11.6 \mathrm{a}$ & $18.5 \mathrm{a}$ & $10.4 \mathrm{a}$ & $22.6 \mathrm{a}$ \\
\hline BRS-Guariba & $2,029 \mathrm{c}$ & $3,516 \mathrm{~b}$ & $1,082 \mathrm{~b}$ & $205.7 \mathrm{a}$ & $44.6 \mathrm{a}$ & $11.1 \mathrm{a}$ & $18.7 \mathrm{a}$ & $12.9 \mathrm{a}$ & $15.6 \mathrm{a}$ \\
\hline BRS-Marataoã & $932 \mathrm{f}$ & $2,748 \mathrm{c}$ & $770 \mathrm{~d}$ & $243.8 \mathrm{a}$ & $40.9 \mathrm{a}$ & $15.3 \mathrm{a}$ & $19.0 \mathrm{a}$ & $13.7 \mathrm{a}$ & $17.4 \mathrm{a}$ \\
\hline BRS-Patativa & $1,616 \mathrm{e}$ & $2,641 \mathrm{c}$ & $1,228 \mathrm{a}$ & $193.4 \mathrm{a}$ & $44.2 \mathrm{a}$ & $11.9 \mathrm{a}$ & $17.3 \mathrm{a}$ & $12.4 \mathrm{a}$ & $19.6 \mathrm{a}$ \\
\hline BRS-Pujante & $1,776 \mathrm{~d}$ & $3,343 \mathrm{~b}$ & $1,045 \mathrm{~b}$ & $182.1 \mathrm{a}$ & $47.3 \mathrm{a}$ & $11.4 \mathrm{a}$ & $20.6 \mathrm{a}$ & $10.2 \mathrm{a}$ & $15.9 \mathrm{a}$ \\
\hline BRS-Rouxinol & $1,248 \mathrm{f}$ & $2,376 \mathrm{~d}$ & $1,117 \mathrm{~b}$ & $295.2 \mathrm{a}$ & $38.7 \mathrm{a}$ & $12.3 \mathrm{a}$ & $20.8 \mathrm{a}$ & $13.3 \mathrm{a}$ & $18.6 \mathrm{a}$ \\
\hline PC951015D01E & $2,165 \mathrm{~b}$ & $4,128 \mathrm{a}$ & $1,145 \mathrm{~b}$ & $199.8 \mathrm{a}$ & $44.6 \mathrm{a}$ & $11.8 \mathrm{a}$ & $18.2 \mathrm{a}$ & $11.8 \mathrm{a}$ & $19.6 \mathrm{a}$ \\
\hline PC950409D02E & $2,177 \mathrm{~b}$ & $4,019 \mathrm{a}$ & $1,051 \mathrm{~b}$ & $165.6 \mathrm{a}$ & $51.4 \mathrm{a}$ & $13.9 \mathrm{a}$ & $18.6 \mathrm{a}$ & $11.9 \mathrm{a}$ & $17.6 \mathrm{a}$ \\
\hline PC951016D01E & $1,340 \mathrm{f}$ & $2,565 \mathrm{c}$ & $686 \mathrm{~d}$ & $154.1 \mathrm{a}$ & $44.2 \mathrm{a}$ & $12.5 \mathrm{a}$ & $17.9 \mathrm{a}$ & $13.2 \mathrm{a}$ & $15.8 \mathrm{a}$ \\
\hline CPCR3F6L15 & $2,400 \mathrm{a}$ & $4,386 \mathrm{a}$ & $1,261 \mathrm{a}$ & $242.9 \mathrm{a}$ & $49.3 \mathrm{a}$ & $13.0 \mathrm{a}$ & $18.7 \mathrm{a}$ & $10.6 \mathrm{a}$ & $21.5 \mathrm{a}$ \\
\hline CPCR3F6L17 & $2,149 \mathrm{~b}$ & $3,850 \mathrm{a}$ & $1,140 \mathrm{~b}$ & $186.3 \mathrm{a}$ & $46.0 \mathrm{a}$ & $12.0 \mathrm{a}$ & $19.0 \mathrm{a}$ & $13.5 \mathrm{a}$ & $18.4 \mathrm{a}$ \\
\hline $\mathrm{C} 1 \mathrm{~J}$ & $1,749 \mathrm{~d}$ & $4,167 \mathrm{a}$ & $751 \mathrm{~d}$ & $169.8 \mathrm{a}$ & $41.5 \mathrm{a}$ & $11.7 \mathrm{a}$ & $18.4 \mathrm{a}$ & $12.7 \mathrm{a}$ & $19.3 \mathrm{a}$ \\
\hline $\mathrm{C} 2 \mathrm{M}$ & $1,716 \mathrm{~d}$ & $4,544 \mathrm{a}$ & $871 \mathrm{c}$ & $306.9 \mathrm{a}$ & $50.0 \mathrm{a}$ & $14.2 \mathrm{a}$ & $20.0 \mathrm{a}$ & $10.8 \mathrm{a}$ & $21.6 \mathrm{a}$ \\
\hline $\mathrm{C} 2 \mathrm{~S}$ & $1,761 \mathrm{~d}$ & $3,101 \mathrm{c}$ & $901 \mathrm{c}$ & $237.0 \mathrm{a}$ & $44.1 \mathrm{a}$ & $12.3 \mathrm{a}$ & $18.9 \mathrm{a}$ & $12.7 \mathrm{a}$ & $19.4 \mathrm{a}$ \\
\hline $\mathrm{C} 3 \mathrm{~F}$ & $1,214 \mathrm{f}$ & $2,909 \mathrm{c}$ & $895 \mathrm{c}$ & $232.7 \mathrm{a}$ & $48.1 \mathrm{a}$ & $11.0 \mathrm{a}$ & $19.3 \mathrm{a}$ & $11.6 \mathrm{a}$ & $21.1 \mathrm{a}$ \\
\hline C3Q & $1,478 \mathrm{e}$ & $2,990 \mathrm{c}$ & $1,012 \mathrm{~b}$ & $240.2 \mathrm{a}$ & $43.6 \mathrm{a}$ & $15.3 \mathrm{a}$ & $15.9 \mathrm{a}$ & $10.1 \mathrm{a}$ & $17.2 \mathrm{a}$ \\
\hline $\mathrm{C} 3 \mathrm{~S}$ & $1,587 \mathrm{e}$ & $3,725 \mathrm{~b}$ & $903 \mathrm{c}$ & $313.4 \mathrm{a}$ & $42.0 \mathrm{a}$ & $13.2 \mathrm{a}$ & $19.7 \mathrm{a}$ & $10.5 \mathrm{a}$ & $19.0 \mathrm{a}$ \\
\hline P290 & $2,141 \mathrm{~b}$ & $4,294 \mathrm{a}$ & $1,148 \mathrm{~b}$ & $235.7 \mathrm{a}$ & $44.3 \mathrm{a}$ & $12.5 \mathrm{a}$ & $19.0 \mathrm{a}$ & $10.9 \mathrm{a}$ & $15.5 \mathrm{a}$ \\
\hline P303 & $2,282 \mathrm{~b}$ & $3,976 \mathrm{a}$ & $1,089 \mathrm{~b}$ & $215.1 \mathrm{a}$ & $51.8 \mathrm{a}$ & $12.0 \mathrm{a}$ & $18.6 \mathrm{a}$ & $12.9 \mathrm{a}$ & $17.0 \mathrm{a}$ \\
\hline P508 & $2,532 \mathrm{a}$ & $4,716 \mathrm{a}$ & $1,351 \mathrm{a}$ & $253.9 \mathrm{a}$ & $40.1 \mathrm{a}$ & $12.0 \mathrm{a}$ & $17.1 \mathrm{a}$ & $11.5 \mathrm{a}$ & $19.5 \mathrm{a}$ \\
\hline PJJ21 & $1,291 \mathrm{f}$ & $2,588 \mathrm{c}$ & $957 \mathrm{c}$ & $178.1 \mathrm{a}$ & $47.3 \mathrm{a}$ & $12.5 \mathrm{a}$ & $18.1 \mathrm{a}$ & $11.6 \mathrm{a}$ & $16.8 \mathrm{a}$ \\
\hline PJM22 & $1,981 \mathrm{c}$ & $3,593 \mathrm{~b}$ & $1,263 \mathrm{a}$ & $229.3 \mathrm{a}$ & $45.4 \mathrm{a}$ & $14.2 \mathrm{a}$ & $20.0 \mathrm{a}$ & $11.2 \mathrm{a}$ & $20.0 \mathrm{a}$ \\
\hline PL23 & $1,301 \mathrm{f}$ & $2,163 d$ & $949 \mathrm{c}$ & $211.5 \mathrm{a}$ & $44.0 \mathrm{a}$ & $12.6 \mathrm{a}$ & $20.8 \mathrm{a}$ & $13.3 \mathrm{a}$ & $19.8 \mathrm{a}$ \\
\hline PAG24 & $1,543 \mathrm{e}$ & $2,677 \mathrm{c}$ & $979 \mathrm{c}$ & $205.9 \mathrm{a}$ & $49.2 \mathrm{a}$ & $11.5 \mathrm{a}$ & $18.9 \mathrm{a}$ & $12.7 \mathrm{a}$ & $19.8 \mathrm{a}$ \\
\hline PC25 & $1,123 \mathrm{f}$ & $1,942 \mathrm{~d}$ & $777 \mathrm{~d}$ & $237.8 \mathrm{a}$ & $41.8 \mathrm{a}$ & $12.0 \mathrm{a}$ & $17.7 \mathrm{a}$ & $10.1 \mathrm{a}$ & $20.6 \mathrm{a}$ \\
\hline PD26 & $1,089 \mathrm{f}$ & $1,783 \mathrm{~d}$ & $950 \mathrm{c}$ & $227.1 \mathrm{a}$ & $45.9 \mathrm{a}$ & $10.9 \mathrm{a}$ & $21.0 \mathrm{a}$ & $12.8 \mathrm{a}$ & $18.8 \mathrm{a}$ \\
\hline PJ27 & $1,698 \mathrm{~d}$ & $3,115 \mathrm{c}$ & $976 \mathrm{c}$ & $238.5 \mathrm{a}$ & $45.0 \mathrm{a}$ & $11.3 \mathrm{a}$ & $19.7 \mathrm{a}$ & $11.3 \mathrm{a}$ & $16.6 \mathrm{a}$ \\
\hline PJJ28 & $1,918 \mathrm{c}$ & $3,452 \mathrm{~b}$ & $881 \mathrm{c}$ & $154.3 \mathrm{a}$ & $46.7 \mathrm{a}$ & $14.4 \mathrm{a}$ & $17.0 \mathrm{a}$ & $12.5 \mathrm{a}$ & $16.2 \mathrm{a}$ \\
\hline PJN29 & $1,741 \mathrm{~d}$ & $2,895 \mathrm{c}$ & $1,137 \mathrm{~b}$ & $274.6 \mathrm{a}$ & $47.8 \mathrm{a}$ & $14.9 \mathrm{a}$ & $18.9 \mathrm{a}$ & $12.1 \mathrm{a}$ & $18.3 \mathrm{a}$ \\
\hline PLP30 & $1,234 \mathrm{f}$ & $2,412 \mathrm{~d}$ & $557 \mathrm{~d}$ & $144.4 \mathrm{a}$ & $45.8 \mathrm{a}$ & $10.6 \mathrm{a}$ & $18.9 \mathrm{a}$ & $13.3 \mathrm{a}$ & $18.7 \mathrm{a}$ \\
\hline MST & $2,682 * *$ & $982,870 * *$ & $421,011 * *$ & $35,354 * *$ & $61.1^{*}$ & $16 * *$ & $25 * *$ & $23 * *$ & $55 * *$ \\
\hline CV $(\%)$ & 32 & 35 & 35 & 41 & 14 & 17 & 11 & 18 & 29 \\
\hline Mean & 1,706 & 3,266 & 1,002 & 222 & 45.4 & 12.6 & 18.8 & 12.0 & 18.6 \\
\hline
\end{tabular}

showed inconsistencies in clusters developed by both methods (Tables 4 and 5).

The cophenetic value by the UPGMA method was 0.69 , indicating limitations in the developed dendrogram. The coefficient of similarity in this dendrogram ranged from 18 to $54 \%$, suggesting a high variability among the cowpea genotypes. The cut-off point at the similarity distance of 0.21 formed seven groups. The genotypes were clustered as group 1 (BRS-Acauã, BRS-Guariba, BRS-Pujante and CPCR3F6L17), group 2 (C3Q), group 3 (C3F, PJM22, C3S, PD26 and PLP30), group 4 (BRSMarataoã, P508, PCCR3F6L15 and C2M), group 5 (the remaining 14 genotypes), group 6 (PJJ21) and group 7 (PC25). The cultivar BRS-Patativa and the line $\mathrm{C} 2 \mathrm{~S}$ showed the highest similarity (54\%), and the genotype PC25 showed the greatest divergence among the evaluated genotypes, justifying its location at the base of the dendrogram (Figure 1).

Due to its genetic variability, high adaptability, yield potential, excellent nutritional value and good behavior, in relation to water deficit, cowpea is considered a valuable crop for semi-arid regions. However, although highly adaptable to the most diverse environments, Santos (2011) reported that cowpea still reaches low dry seed yields, estimated at around $300 \mathrm{~kg} \mathrm{ha}^{-1}$ in northeastern Brazil. According to Silva et al. (2010), climatic 
Table 4. Number and identification of thirty cowpea genotypes [Vigna unguiculata (L.) Walp] evaluated for eight qualitative and nine agronomic variables and distributed into three groups according to the k-means clustering method.

\begin{tabular}{ccl}
\hline Group & Number of genotypes & \multicolumn{1}{c}{ Genotypes } \\
\hline 1 & 3 & C3F, C3S, PD26 \\
& & BRS-Guariba, BRS-Marataoã, BRS-Patativa, BRS-Pujante, BRS-Rouxinol, PC951015D01E, \\
2 & 24 & $\begin{array}{l}\text { PC950409D02E, PC951016D01E, CPCR3F6L15, CPCR3F6L17, C1J, C2M, C2S, P-290, P-303, } \\
\text { P-508, PJJ21, PJM22, PL23, PAG24, PJ27, PJJ28, PJN29, PLP30 } \\
3\end{array}$ \\
\hline
\end{tabular}

Table 5. Number and identification of thirty cowpea genotypes [Vigna unguiculata (L.) Walp] evaluated for eight qualitative and nine agronomic variables and distributed into six groups according to the Tocher clustering method based on the Gower algorithm.

\begin{tabular}{ccl}
\hline Group & Number of genotypes & \multicolumn{1}{c}{ Genotypes } \\
\hline & & PC951015D01E, C1J, C2S, PJJ28, BRS-Guariba, CPCR3F6L17, P-303, PAG24, BRS-Patativa, \\
1 & 19 & $\begin{array}{l}\text { PJ27, PC951015D01E, P-290, PC950409D02E, PL23, PJM22, BRS-Pujante, BRS-Rouxinol, } \\
\text { PJN29, PLP30 } \\
2\end{array}$ \\
3 & 3 & C3F, C3S, PD26 \\
4 & 3 & CPCR3F6L15, C2M, BRS-Acauã \\
5 & 2 & C3Q, PJJ21 \\
6 & 2 & BRS-Marataoã, P-508 \\
\end{tabular}

adversities, poor agricultural technology and the use of unimproved seeds are the leading causes of low yields in the semi-arid region of Brazil. Moreover, the growing conditions of different environments may result in unequal genotype performances. In order to estimate the phenotypic variability, the comprehensive evaluation of cowpea genotypes intended for immature seed harvesting is a crucial step toward developing new cowpea cultivars by crossing divergent genotypes with good agronomic performance, as proposed in this study.

Cowpea production for immature seed or pod commercialization is often carried out by farmers who prefer genotypes with an indeterminate growth habit and uneven maturation, resulting in several harvests during the crop cycle and high yields. The consumer preference is predominantly for easily cooked, purple and immature pods and seeds with good taste and pleasant smell. Moreover, traders prefer genotypes that are easy to thresh and with a good post-harvest quality (Rocha et al. 2006).

The overall average of plant growth habit among the genotypes was 1.90 , which was similar to the average found by Rocha et al. (2006) (1.93). The overall average of the cultivation value for the tested genotypes was 2.18 , higher than the average found by Andrade et al. (2010) (1.89). The overall average of pod length for the tested genotypes was
$20.1 \mathrm{~cm}$, similar to the value reported by Andrade et al. (2010) (19.63 cm).

The overall average of 100-immature seed weight was 45.4 , above the weight required by the immature seed market $(30 \mathrm{~g})$. This average was also higher than that obtained by Rocha et al. (2006) (36 g) and Andrade et al. (2010) (37 g). According to Rocha et al. (2007), genotypes for immature seed production include a longer flowering period, high pod yield, attractive and well-seeded pods, immature seed to pod weight ratio over $60 \%$ and seeds capable of preserving good post-harvest and post-threshing aspects. In this scenario, fast darkening seeds are not well received by the market.

The number of groups differed according to the three applied methods, with the k-means clustering resulting in the smallest number of groups, since, in this method, there is a previously defined number of groups. The same discrepancies were observed for the accessions, except for C3F, C3S and PD26, which were consistently grouped by the k-means (group 1) and Tocher (group 2) methods. Another exception was observed for the accession PC25, consistently grouped by the Tocher method and the dendrogram based on the Jaccard coefficient.

Based on two clustering validation methods (Dunn and DB index), Adarsh et al. (2020) concluded that the non-hierarchical k-means clustering was 


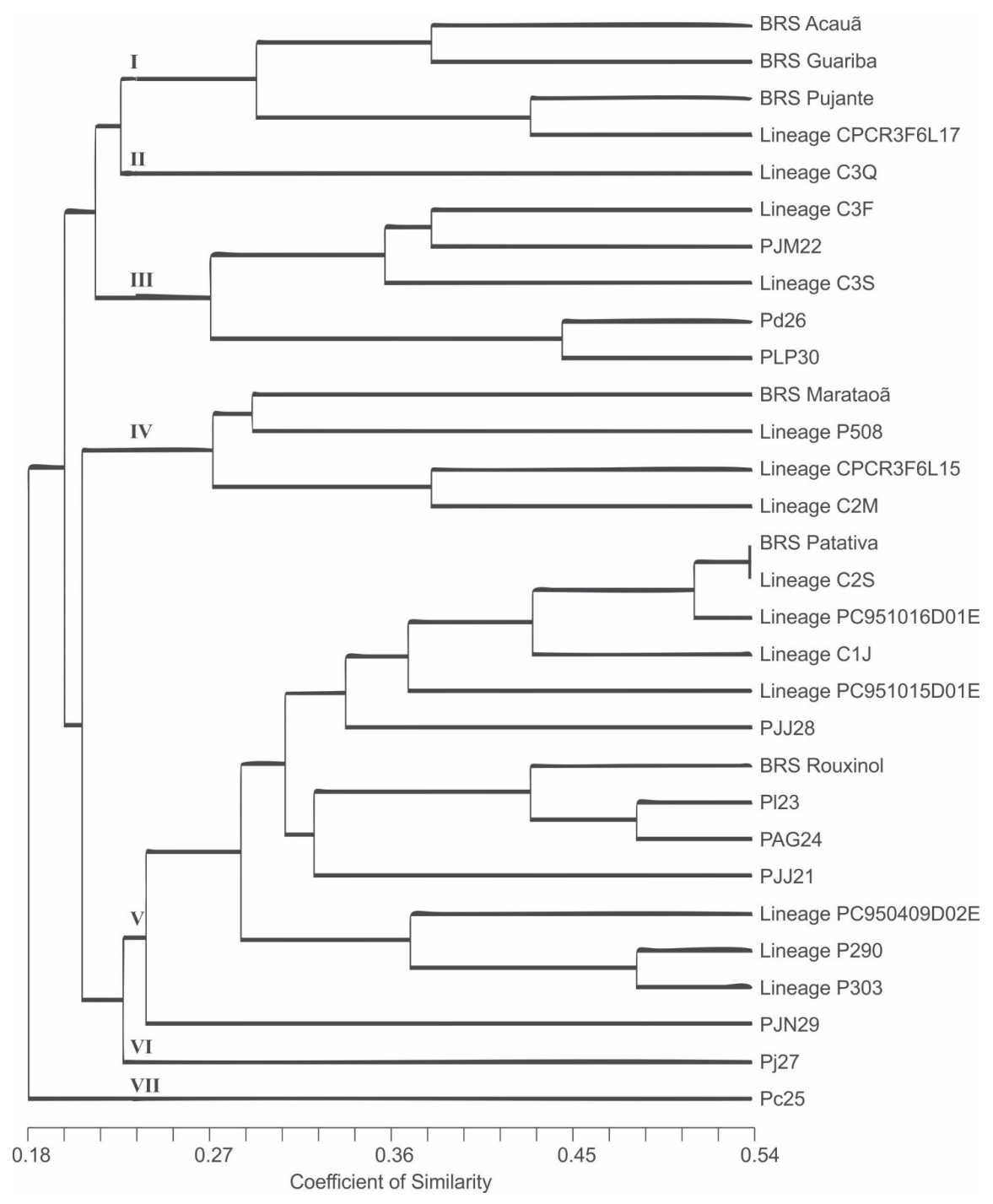

Figure 1. Unweighted pair group method with arithmetic mean dendrogram obtained from the evaluation of seventeen traits in thirty cowpea genotypes [Vigna unguiculata (L.) Walp]. Cophenetic value $=0.69$.

superior to the Tocher method and other hierarchical methods adopted in the present study, e.g., UPGMA. The criticism against the $\mathrm{k}$-means clustering is because the number of clusters is defined before the analysis (Cruz et al. 2004), which could occur based on some biological evidence, as adopted in the present study.

Despite the preference for commercialization as immature seeds, there are no cowpea cultivars identified for this purpose, and studies on the evaluation of cowpea accessions for breeding programs are scarce. The thirty cowpea genotypes showed a high phenotypic variability for the agronomic traits evaluated, highlighting their potential for breeding programs involving immature seed harvesting in the near future.
The genotypes P508 and PCCR3F6L15 stood out with the highest immature seed yields, a harvesting period of about 20 days and cooking times of 12 and $13 \mathrm{~min}$, respectively. They also had semierect to prostrate plant growth habits, easily opened pods and easy seed release by manual threshing. Nonetheless, these two genotypes showed dark seeds at $48 \mathrm{~h}$ after threshing, which is undesirable for the commercialization of immature seeds.

The genotype BRS-Acauã showed light-dark seeds at $48 \mathrm{~h}$ after threshing, with an immature seed yield of 2,000 $\mathrm{kg} \mathrm{ha}^{-1}$ and a harvesting period of more than 22 days. Moreover, this genotype was clustered into different groups based on the cluster analysis conducted in this study. Therefore, BRS-Acauã is a 
good option for crosses with P508 and PCCR3F6L15 to develop new immature seed lines.

\section{CONCLUSIONS}

1. The thirty cowpea genotypes showed statistically significant differences $(\mathrm{p}<0.05)$, suggesting a high phenotypic variability for all the agronomic variables evaluated for immature seed harvesting;

2. The number of groups differed according to the three methods applied, with the k-means clustering resulting in the smallest number of groups. The accession groups showed discrepancies, with only two grouping exceptions;

3. The accessions BRS Acauã, in one group, and P508 and PCCR3F6L15, in other groups, showed a good quality for immature grain harvest and were consistently grouped into different clusters by the three methods applied. These accessions are recommended as parents to develop new cultivars.

\section{ACKNOWLEDGMENTS}

The authors would like to thank the Coordenação de Aperfeiçoamento de Pessoal de Nível Superior (Capes), for the scholarship granted.

\section{REFERENCES}

ADARSH, V. S.; JOSEPH, B.; GOPINATH, P. P. Comparison of clustering techniques used in divergence analysis. International Journal of Statistics and Applied Mathematics, v. 5, n. 4, p. 19-21, 2020.

ANDRADE, F. N. Avaliação e seleção de linhagens de tegumento e cotilédone para o mercado de feijão-caupi verde. 2010. Dissertação (Mestrado em Agronomia) Universidade Federal do Piauí, Teresina, 2010.

ANDRADE, F. N.; ROCHA, M. M.; GOMES, R. L. F.; FREIRE FILHO, F. R.; RAMOS, S. R. R. Estimativas de parâmetros genéticos em genótipos de feijão-caupi avaliados para feijão fresco. Agronomic Science Magazine, v. 41, n. 2, p. 253-258, 2010.

CRUZ, C. D. Genes software: extended and integrated with the R, Matlab and Selegen. Version 2015.5.0. Acta Scientiarum, v. 38, n. 4, p. 547-552, 2016.

CRUZ, C. D.; REGAZZI, A. J.; CARNEIRO, P. C. S. Modelos biométricos aplicados ao melhoramento genético. 4. ed. Viçosa: UFV, 2004.
ROCHA, M. M.; FREIRE FILHO, F. R.; RAMOS, S. R. R.; RIBEIRO, V. Q.; ANDRADE, F. N.; GOMES, R. L. F. Avaliação agronômica de genótipos de feijão-caupi para produção de grãos verdes. Teresina: Embrapa Meio-Norte, 2006. (Boletim de pesquisa e desenvolvimento, 67).

ROCHA, M. M.; FREIRE FILHO, F. R.; RIBEIRO, V. Q.; CARVALHO, H. W. L. C.; BELARMINO FILHO, J.; RAPOSO, J. A. A.; ALCÂNTARA, J. P.; RAMOS, S. R. R.; MACHADO, C. F. Adaptabilidade e estabilidade produtiva de genótipos de feijão-caupi de porte semiereto na região Nordeste do Brasil. Pesquisa Agropecuária Brasileira, v. 42, n. 9, p. 1283-1289, 2007.

ROHLF, F. J. NTSYS-pc numerical taxonomy and multivariate analysis system. Version 2.1. New York: Exeter, 2000.

SANTOS, C. A. F. Cultivares de feijão-caupi para o Vale do São Francisco. Petrolina: Embrapa Semiárido, 2011. (Circular técnica, 94).

SAS INSTITUTE. SAS/STAT user's guide. Version 6. Cary: SAS Institute, 1989.

SILVA FILHO, A. J. R.; ANTONIO, R. P.; SILVA, P. S. L.; SILVEIRA, L. M.; ALBURQUERQUE, L. B. Avaliação morfológica e agronômica de sementes de acessos de caupi coletados no Rio Grande do Norte. Agropecuária Científica no Semiárido, v. 9, n. 4, p. 102-106, 2013.

SILVA, V. P. R.; CAMPOS, J. H. B. C.; SILVA, M. T.; AZEVEDO, P. V. Impact of global warming on cowpea bean cultivation in northeastern Brazil. Agricultural Water Management, v. 97, n. 11, p. 1760-1768, 2010.

SOUSA, J. L. M.; ROCHA, M. de M.; SILVA, K. J. D. e; NEVES, A. C. das; SOUSA, R. R. de. Potencial de genótipos de feijão-caupi para o mercado de vagens e grãos verdes. Pesquisa Agropecuária Brasileira, v. 50, n. 5, p. 392-398, 2015.

TEIXEIRA, I. R.; SILVA, G. C.; OLIVEIRA, J. P. R.; SILVA, A. G.; PELÁ, A. Desempenho agronômico e qualidade de semente de cultivares de feijão-caupi na região do Cerrado. Revista Ciência Agronômica, v. 41, n. 2, p. 300-307, 2010.

VENCOVSKY, R.; BARRIGA, P. Genética biométrica no fitomelhoramento. Ribeirao Preto: Sociedade Brasileira de Genética, 1992. 